\title{
Effectiveness of valproate for the treatment of manic-like behavior in X-linked adrenoleukodystrophy
}

\author{
Ettore Salsano • Orsola Gambini • \\ Anna Rita Giovagnoli • Laura Farina • \\ Graziella Uziel · Davide Pareyson
}

Received: 11 November 2011/Accepted: 15 November 2011/Published online: 27 November 2011

(c) Springer-Verlag 2011

Keywords Adrenoleukodystrophy · Disinhibition · Irritability · Mania · Valproate

Dear Editor,

A 24-year-old male with cerebral X-linked adrenoleukodystrophy (X-ALD) due to the N148S mutation in ABCD1 was admitted because of worsening behavioral changes and appearance of mild gait instability. Disease onset occurred at the age of 14, with irritability, aggressiveness, adaptive difficulties and learning disabilities. He also suffered from adrenal insufficiency on replacement therapy since age 14 . At neurological examination, he presented minimal gait ataxia, slight gaze-evoked nystagmus, mild intentional tremor of the left arm, and brisk deep tendon reflexes. He also

E. Salsano · D. Pareyson

SOSD Clinica delle Neuropatie Degenerative Centrali e Periferiche, Fondazione IRCCS, Istituto Neurologico C. Besta, Milan, Italy

O. Gambini

DMCO, Università degli Studi di Milano, AO San Paolo, Milan, Italy

A. R. Giovagnoli

SOSD Neuropsicologia Clinica, Fondazione IRCCS,

Istituto Neurologico C. Besta, Milan, Italy

L. Farina

UO Neuroradiologia, Fondazione IRCCS, Istituto Neurologico

C. Besta, Milan, Italy

G. Uziel $(\bowtie)$

SOSD Malattie Genetiche Metaboliche e Degenerative, Fondazione IRCCS, Istituto Neurologico C. Besta,

via Celoria 11, 20133 Milan, Italy

e-mail: graziella.uziel@istituto-besta.it appeared irritable, restless, distractible, disinhibited, loudly speaking, perseverant, self-neglectful and with poor impulse control. He received a diagnosis of manic-like behavioral disturbance associated with cognitive impairment. Manic symptoms were quantified with the 11-item Young Mania Rating Scale (YMRS) [1], whereas the cognitive impairment was evaluated by formal neuropsychological tests (Table 1). YMRS score was 15/56, and the patient showed a severe impairment of visuomotor coordination, divided attention, executive functions, and verbal learning and delayed recall, suggesting a predominant frontal lobe dysfunction. Brain MRI demonstrated X-ALD-related abnormalities (Fig. 1). We decided to start treatment with valproate $1,000 \mathrm{mg}$ bid, as valproate acts also as a mood-stabilizing agent. After 6 months, the patient was re-assessed and we observed a relevant improvement of his manic-like behavioral disturbances as well as of his cognitive performances (Table 1). In particular, with respect to baseline assessment, we registered a YMRS score reduction, and an improvement of his attentive, executive, and memory abilities. We remark that the features of mania, including disinhibition, hypersexuality, and irritability, often followed by cognitive decline, may be the first clinical manifestations of the adolescent cerebral $\mathrm{X}$-ALD, as occurs for the adult cerebral forms [2]. From a therapeutic perspective, we note that in patients with organic psychiatric disorders, such as cerebral X-ALD, the appropriate treatment of psychiatric symptoms may be problematic, as they often do not respond to medications in a typical manner [2]. Moreover, given the rarity of X-ALD and the severity of its course, high quality studies dealing with symptomatic therapies are difficult to realize. The treatment of psychiatric symptoms, however, should not be neglected, as cerebral X-ALD is a progressive disease with no causative therapy, and imposes a high burden of care to the patient's families. Lithium, benzodiazepines and even neuroleptics 
Table 1 Neuropsychological tests and Young Mania Rating Scale (YMRS) in a case of adolescence cerebral X-adrenoleukodystrophy

\begin{tabular}{|c|c|c|c|}
\hline Cognitive domains & Neuropsychological tests & $\begin{array}{l}\text { Before valproate } \\
\text { (raw score; equivalent score }^{\mathrm{a}} \text { ) }\end{array}$ & $\begin{array}{l}\text { Under valproate } \\
\text { (raw score; equivalent score }{ }^{a} \text { ) }\end{array}$ \\
\hline Young Mania Rating Scale (YMRS) & & $15 / 56$ & $5 / 56$ \\
\hline \multicolumn{4}{|l|}{ Attention } \\
\hline Selective attention & Attentive matrices & $49 / 60 ; 2$ & $49 / 60 ; 2$ \\
\hline Visuo-motor coordination & Trial Making Test A & $94^{\prime \prime} ; 0$ & $70^{\prime \prime} ; 1$ \\
\hline Divided attention & Trial Making Test B & $275^{\prime \prime} ; 0$ & $150^{\prime \prime} ; 2$ \\
\hline \multicolumn{4}{|l|}{ Executive functions } \\
\hline & Frontal Assessment Battery & $14 / 18 ; 0$ & $15 / 18 ; 1$ \\
\hline & Phonemic fluency & $15 ; 0$ & $12 ; 0$ \\
\hline Planning & Tower of London & $19 / 36$ (cut off $<26,54)$ & $28 / 36$ (cut off $<26,54)$ \\
\hline Mental flexibility & Weigl's Sorting Test & $8 / 15 ; 1$ & $13 / 15 ; 4$ \\
\hline \multicolumn{4}{|l|}{ Memory and learning } \\
\hline & Digit span & $5 ; 3$ & $6 ; 4$ \\
\hline & Short story recall & $7 / 28 ; 0$ & $11 / 28 ; 2$ \\
\hline Verbal learning & RAVLT-immediate & $27 / 75 ; 0$ & $43 / 75 ; 2$ \\
\hline Long-term memory & RAVLT-delayed & $3 ; 0$ & $7 ; 0$ \\
\hline \multicolumn{4}{|l|}{ Lexical-semantic abilities } \\
\hline & Word fluency on semantic cue & $28 ; 1$ & $26 ; 0$ \\
\hline & Token Test & 31,$5 ; 2$ & ND \\
\hline Visuospatial abilities & Street Completion Test & $10 ; 4$ & ND \\
\hline \multicolumn{4}{|l|}{ Praxes } \\
\hline & Imitating gestures & $20 ; 4$ & ND \\
\hline & Imitating facial expression & $20 ; 4$ & ND \\
\hline
\end{tabular}

${ }^{a}$ Equivalent scores are derived from the normative data of the Italian population. The score 0 corresponds to a pathological performance, the score 1 corresponds to the lower normal limit, while 2, 3, and 4 are all scores in the normal range

RAVLT Rey Auditory Verbal Learning Test
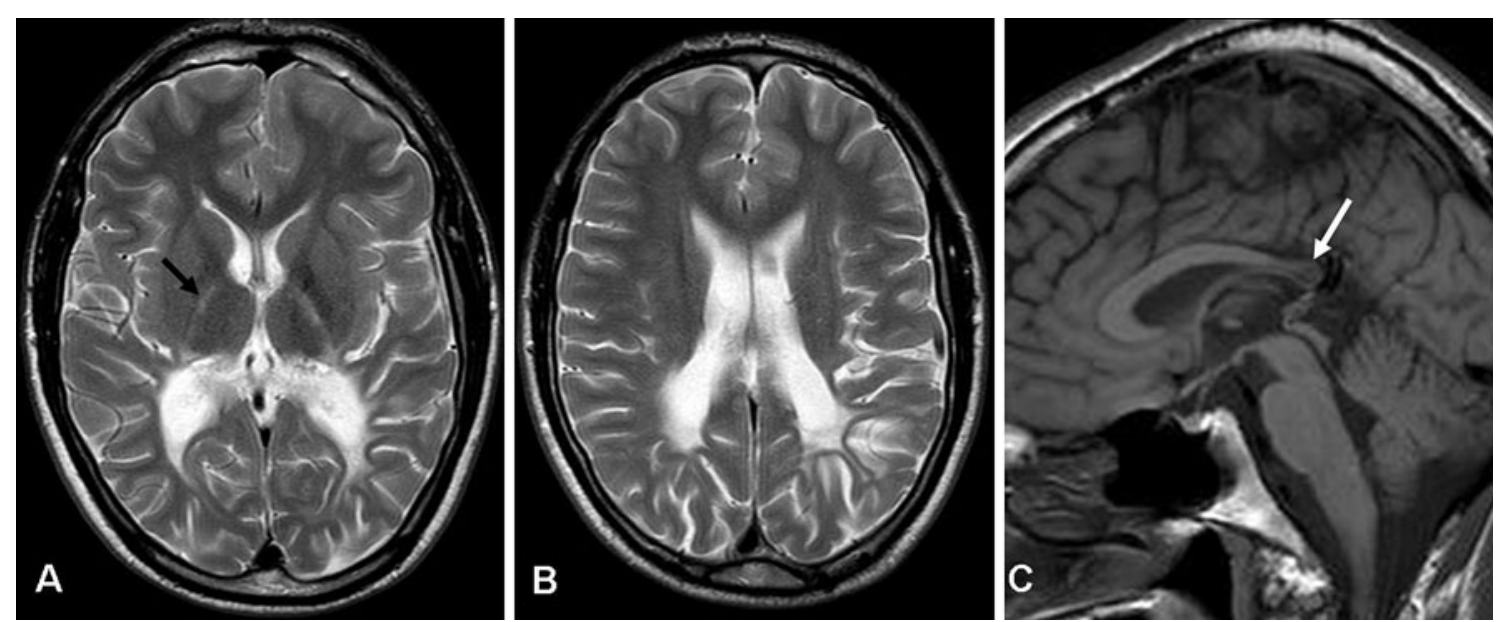

Fig. 1 Brain MRI of a case of adolescent cerebral X-ALD. Axial T2weighted images $(\mathbf{a}, \mathbf{b})$ show hyperintensity of the posterior periventricular white matter, and enlargement of the posterior horns of the lateral ventricles and parieto-occipital sulci; hyperintensity of

have been considered for the treatment of manic-like behavioral changes of X-ALD patients, but no information is available whether one drug is more effective and better the posterior internal capsules is also present (a, black arrow). Midline sagittal T1-weighted image (c) shows atrophy and signal abnormality of the splenium (white arrow)

tolerated than another [2]. Here, we have found that valproate was well-tolerated and effective in improving the manic-like symptoms in a patient with adolescent cerebral 
$\mathrm{X}$-ALD, as previously observed in a case of adult cerebral $\mathrm{X}$-ALD with behavior and mood disturbances [3]. Interestingly, we also noted a marked improvement of the patient's cognitive profile, suggesting that the results of neuropsychological tests in patients with cerebral X-ALD and predominantly frontal lobe symptoms should be cautiously interpreted when psychiatric manifestations are present. Such a cognitive improvement supports a role for the socalled 'psychopathological interference'. Accordingly, in the present case, leukodystrophy was predominant in the posterior white matter, which, unlike frontal-subcortical damage, is usually not a cause of executive dysfunctions. Valproate has always been considered a safe drug; moreover, valproic acid was recently found to reduce the oxidative damage to proteins in X-ALD patients, indicating that it might be a novel disease-modifying therapy for X-ALD [4]. Unlike valproate, lithium has a low margin of safety, especially in patients with adrenal failure, as are those with $\mathrm{X}$-ALD. Indeed, long-term lithium therapy inhibits the action of aldosterone on the distal tubule of the kidney [5]; this, in turn, leads to the increase of aldosterone secretion in healthy subjects, but not in patients with adrenal failure, thus favoring hypotension and plasma electrolyte disturbances (e.g., hyponatremia) [6]. Neuroleptics are responsible for many side effects, including parkinsonism, which may further impair the ability of X-ALD patients to move about, and they might promote the formation of reactive oxygen species in the brain, thus favoring disease progression [2, 7]. Finally, long-term treatment with benzodiazepines can worsen ataxia, and cause dependence with withdrawal side effects, such as agitation, irritability, and aggressive behavior [2]. In conclusion, our observation encourages to consider valproate as first-choice therapy for the manic-like behavioral disturbances in X-ALD patients.

\section{References}

1. Young RC, Biggs JT, Ziegler VE, Meyer DA (1978) A rating scale for mania: reliability, validity and sensitivity. $\mathrm{Br} \mathrm{J}$ Psychiatry 133:429-435

2. Rosebush PI, Garside S, Levinson AJ, Mazurek MF (1999) The neuropsychiatry of adult-onset adrenoleukodystrophy. J Neuropsychiatry Clin Neurosci 11:315-327

3. Leo RJ (1998) Behavioral changes and affective instability associated with adult-onset adrenoleukodystrophy. Psychosomatics 39:176-177

4. Fourcade S, Ruiz M, Guilera C et al (2010) Valproic acid induces antioxidant effects in X-linked adrenoleukodystrophy. Hum Mol Genet 19:2005-2014

5. Thomsen K, Bak M, Shirley DG (1999) Chronic lithium treatment inhibits amiloride-sensitive sodium transport in the rat distal nephron. J Pharmacol Exp Ther 289:443-447

6. Stewart PM, Grieve J, Nairn IM, Padfield PL, Edwards CR (1987) Lithium inhibits the action of fludrocortisone on the kidney. Clin Endocrinol (Oxf) 27:63-68

7. Heiser P, Sommer O, Schmidt AJ et al (2010) Effects of antipsychotics and vitamin $\mathrm{C}$ on the formation of reactive oxygen species. J Psychopharmacol. 24:1499-1504 